\title{
Cloud's Center of Gravity - a compact approach to analyze convective cloud development
}

\author{
I. Koren ${ }^{1}$, O. Altaratz ${ }^{1}$, G. Feingold ${ }^{2}$, Z. Levin ${ }^{3}$, and T. Reisin ${ }^{4}$ \\ ${ }^{1}$ Dept. of Environ. Sciences Weizmann Institute, Rehovot, Israel \\ ${ }^{2}$ NOAA Earth System Research Laboratory, Boulder, Colorado, USA \\ ${ }^{3}$ Dept. of Geophysics and Planetary Sciences, Tel Aviv University, Tel Aviv, Israel \\ ${ }^{4}$ Soreq Nuclear Research Center, Yavne, Israel \\ Received: 14 May 2008 - Published in Atmos. Chem. Phys. Discuss.: 23 July 2008 \\ Revised: 20 October 2008 - Accepted: 20 October 2008 - Published: 12 January 2009
}

\begin{abstract}
As cloud resolving models become more detailed, with higher resolution outputs, it is often complicated to isolate the physical processes that control the cloud attributes. Moreover, due to the high dimensionality and complexity of the model output, the analysis and interpretation of the results can be very complicated. Here we suggest a novel approach to convective cloud analysis that yields more insight into the physical and temporal evolution of clouds, and is compact and efficient. The different (3-D) cloud attributes are weighted and projected onto a single point in space and in time, that has properties of, or similar to, the Center Of Gravity (COG). The location, magnitude and spread of this variable are followed in time. The implications of the COG approach are demonstrated for a study of aerosol effects on a warm convective cloud. We show that in addition to reducing dramatically the dimensionality of the output, such an approach often enhances the signal, adds more information, and makes the physical description of cloud evolution clearer, allowing unambiguous comparison of clouds evolving in different environmental conditions. This approach may also be useful for analysis of cloud data retrieved from surface or space-based cloud radars.
\end{abstract}

\section{Introduction}

The effect of aerosol on clouds and precipitation poses the largest uncertainty in the estimation of the anthropogenic contribution to climate change (IPCC, 2007; Levin and Cotton, 2007). However due to the sparse distribution and short

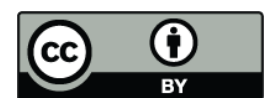

Correspondence to: I. Koren (ilan.koren@weizmann.ac.il) lifetime of aerosol, the inherent complexity of cloud microphysics and dynamics, and the strong coupling with meteorology, it is challenging to estimate the overall effect.

Although observations and in-situ measurements provide direct evidence of physical phenomena, they cannot provide a comprehensive description of processes and their feedbacks, due to lack of information in time and/or in space. Models, provided they adequately resolve physical processes and their couplings, are the main tool with which all the information can be integrated, and with which the effects of aerosol can be studied from the microphysical to the whole-cloud dynamical scale. Cloud-resolving numerical models are probably the only tool that can separate causeand-effect and give a more complete physical interpretation of the observed correlations. However such analyses may require many simulations and intensive statistical analysis (e.g., Teller and Levin 2008). The capacity of numerical models is improving significantly, as computers become more powerful. Today, with clusters of many CPUs, models representing many physical variables can be run at high spatial and temporal resolution over large domains. However, a barrier that limits the full potential for progress is that the huge output is often not easy to interpret and sometimes the physical meaning of the results is lost in the large and detailed dimensionality.

Here we propose a compact way to analyze cloud model output in a way that reduces the dimensionality but preserves and emphasizes the physical properties of the cloud. This method adds important insight into cloud evolution and allows efficient comparison of clouds evolving under different conditions. The potential of this method to extract new (often less intuitive) insight into the microphysical and dynamical processes in clouds is demonstrated here for a case study

Published by Copernicus Publications on behalf of the European Geosciences Union. 
of aerosol effects on a single cloud. A demonstration of the strength of this method for analysis of multi-cloud cases (a cloud field) will follow as a sequel to this paper.

\section{Theory - The cloud center of gravity}

Clouds can be described as inhomogeneous clusters of water droplets and ice particles. While condensing and freezing, the hydrometeors release latent heat that further enhances the internal updrafts. This process is opposed by the drag due to the condensed mass and by cooling of the air due to evaporation, sublimation, and melting of precipitation. The basic parameter that reflects the physical state of the cloud is the water/ice content of the different particles. At every given point in time, the distributions of the masses, along with the velocity field reflect the microphysical and dynamical processes affecting the cloud. Such parameters are often described by contours showing the instantaneous structure of the cloud. In other cases, the time variation of the mean or extreme values is presented.

Here we calculate the cloud center of gravity (COG), and a series of statistical moments derived from it. We use the location, magnitude, and spread of these variables to trace the cloud development and to represent compactly the cloud size, and the cloud microphysical and dynamical states, without using extreme values and thresholds. Together with the scalar value (the mass), a single point in space is used to represent the location of the equivalent cloud. A set of three additional numbers is used to represent the distribution of mass inside the 3-D cloud (the spread). We introduce a COG operator that can map any other cloud variable, such as velocity and droplet radius, by similar representations of amplitude (scalar, or vector for winds), a COG-like location point, and a spread. This approach is, by definition, highly sensitive to the cloud microphysical and dynamical state, i.e., the magnitude and distribution of the variables. It can be used, therefore, as an additional tool to gain insight into the physical processes governing the cloud evolution.

The center of gravity $R$ of a system is the point in space (i.e., the physical location) at which the total mass can be considered to concentrate, and at which external forces may be applied. It can also be defined as the average coordinates $R$ of the system elements $r_{i}$ weighted by their masses $m_{i}$ :

$R=\frac{\sum_{i} r_{i} m_{i}}{M}$,

where the total mass of condensate $M$ of the system is

$M=\sum_{i} m_{i}$
$R$ is commonly used in classical and quantum mechanics in order to solve complex problems of many-body systems (Feynman, 1963). In this paper, the approach will be described for a single cloud but it can be applied in a similar way to a cloud field.

We will define the spread $S$ of the cloud (in any spatial dimension $x, y$, or $z$ ) as the distance from $R$ weighted by mass, or the weighted standard deviation of the distances from the center of gravity:

$S=\sqrt{\frac{\sum_{i} m_{i}\left(r_{i}-R\right)^{2}}{M}}$.

$S$ provides a statistically robust measure of the variance around the COG of the cloud properties.

Using the horizontal spreads along the $\mathrm{x}$ and $\mathrm{y}$ axes $\left(\mathrm{S}_{x}\right.$ and $\mathrm{S}_{y}$ ), we will define the effective area $A$ :

$A=S_{x} S_{y}$

Or the effective aspect ratio $\Phi$ as the ratio between the horizontal to the vertical spread:

$\Phi=\frac{S_{x}}{S_{z}}$

of any cloud property as a measure of the properties' horizontal variance around the COG, the cloud fraction and the structure of the cloud.

When analyzing other cloud attributes it is often informative to see their value weighted by the particle mass. For example the time variations of the vertical velocities weighted by mass will clearly show if the cloud is ascending or descending. Therefore, we define in a similar manner a set of operators of any physical quantity $q$. To do so, we define the momentum-like product $M_{Q}$ of the mass and the variable $q$ as

$M_{Q}=\sum_{i} q_{i} m_{i}$,

and the weighted-by-mass averaging operator $Q$ of the quantity $q$ will be

$Q=\frac{\sum_{i} q_{i} m_{i}}{M}$.

Analogously, the center of gravity operator $R_{Q}$ of the quantity $q$ is defined as

$R_{Q}=\frac{\sum_{i} r_{i} m_{i} q_{i}}{M_{Q}}$

and the spread operator $S_{Q}$ as

$S_{Q}=\sqrt{\frac{\sum_{i} m_{i} q_{i}\left(r_{i}-R\right)^{2}}{M_{Q}}}$. 

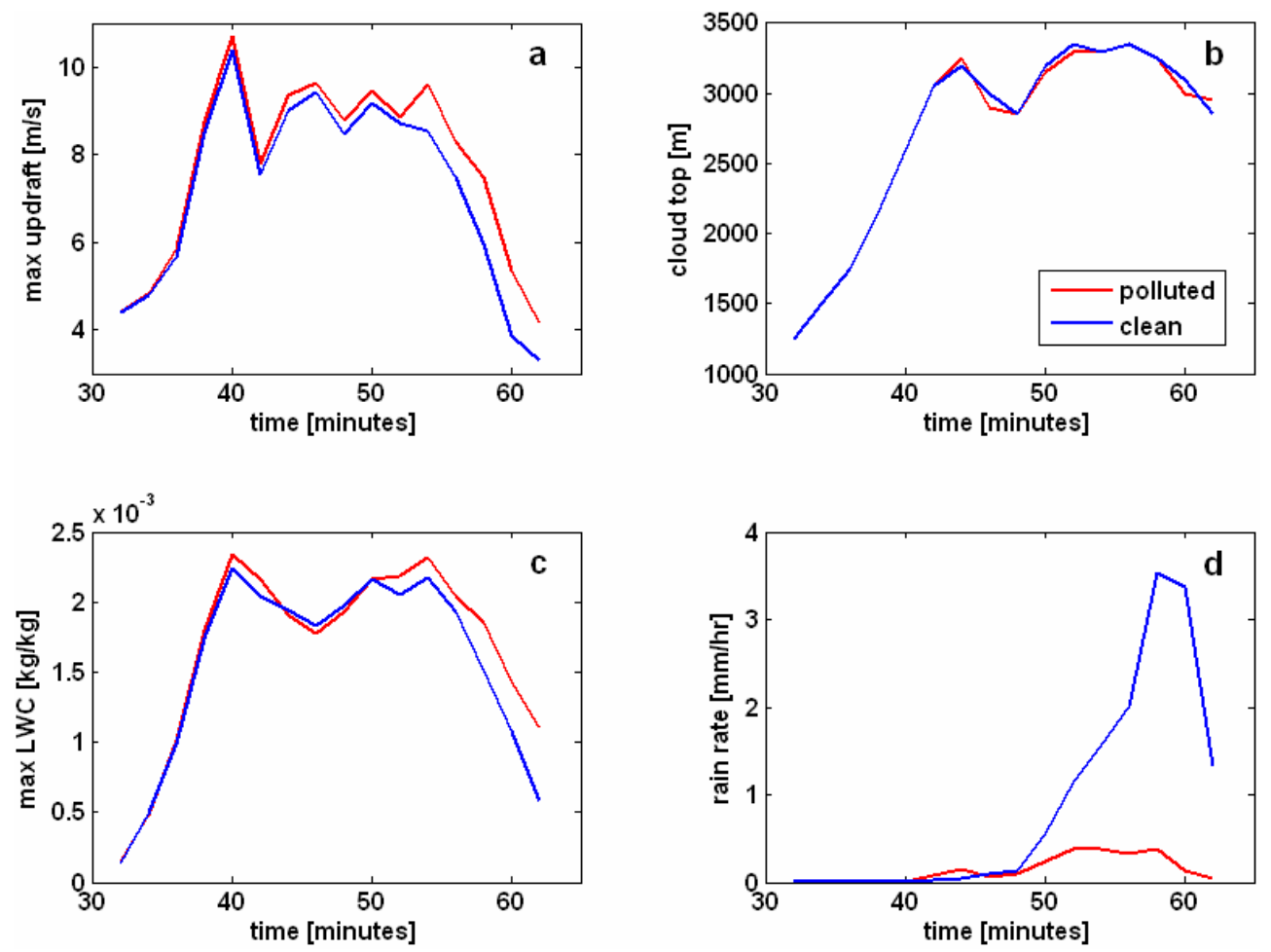

Fig. 1. Basic description of the clouds (blue-clean, red-polluted): (a) - (top left) time evolution of the maximal updraft. (b) (top right), time evolution of the cloud top height. (c) (bottom left), evolution of the clouds maximum LWC and (d) (bottom right) maximal ground rain rate.

Using a similar format to the mass information, these operators can provide insight into the development of other cloud parameters. The development of the weighted-by-mass averages in time and in space (location and spread) will give a stable measure of the evolution of the cloud and facilitate the comparison of clouds evolving in different dynamical and microphysical environments.

The set of the total mass $M$, the center of gravity $R(x, y, z)$ and the spread $S(x, y, z)$ (7 numbers in 3-D cases) provides an abstract measure of the multi-dimensional mass distribution inside the cloud. Likewise, each of the dynamical and microphysical properties (updrafts, effective radius) will be measured by 7 numbers per time step. Therefore, a few numbers ( 7 for each variable) that give a compact measure of the evolution of the clouds replace much of the information in the complete 3-D dataset.

\section{Case study: aerosol effects on a warm convective cloud}

The strength of the COG analysis will be demonstrated with a case study of the interaction of aerosol with a warm convective cloud. A relatively simple example has been chosen to demonstrate the new insight that can be gained by this method.
The RAMS cloud resolving model version 4.3 (Cotton et al., 2003) was used to simulate the development of a small cumulus cloud. The cloud microphysical parameterization is based on a bulk microphysical scheme. For the current simulations, we consider warm, ice-free clouds. The water class is categorized into three different forms: vapor, cloud droplets and rain. The microphysical processes included in the model are activation of droplets, condensation, evaporation, collision and coalescence, collisional breakup, and sedimentation. The microphysical model uses a combination of one or two-moment hydrometeor prediction scheme. A detailed description of the RAMS microphysical model is given in Walko et al. (1995, 2000) and Meyers et al. (1997). The microphysical model was configured in the following manner: the mixing ratio of cloud water was prognosed and the number concentration of cloud droplets specified at either $200 \mathrm{~cm}^{-3}$ (clean) or $1600 \mathrm{~cm}^{-3}$ (polluted). The mixing ratio and number concentration of the raindrops were prognosed using the two-moment scheme.

Two clouds were simulated under the same environmental conditions with different specified cloud droplet number concentrations representing different aerosol loadings. The simulations were initialized with a sounding of temperature and moisture from Bet Dagan, Israel, meteorological station on 12 July 2002 at $12 Z$ (15:00 LT). The profile was modified to include additional moisture at the low levels for a 

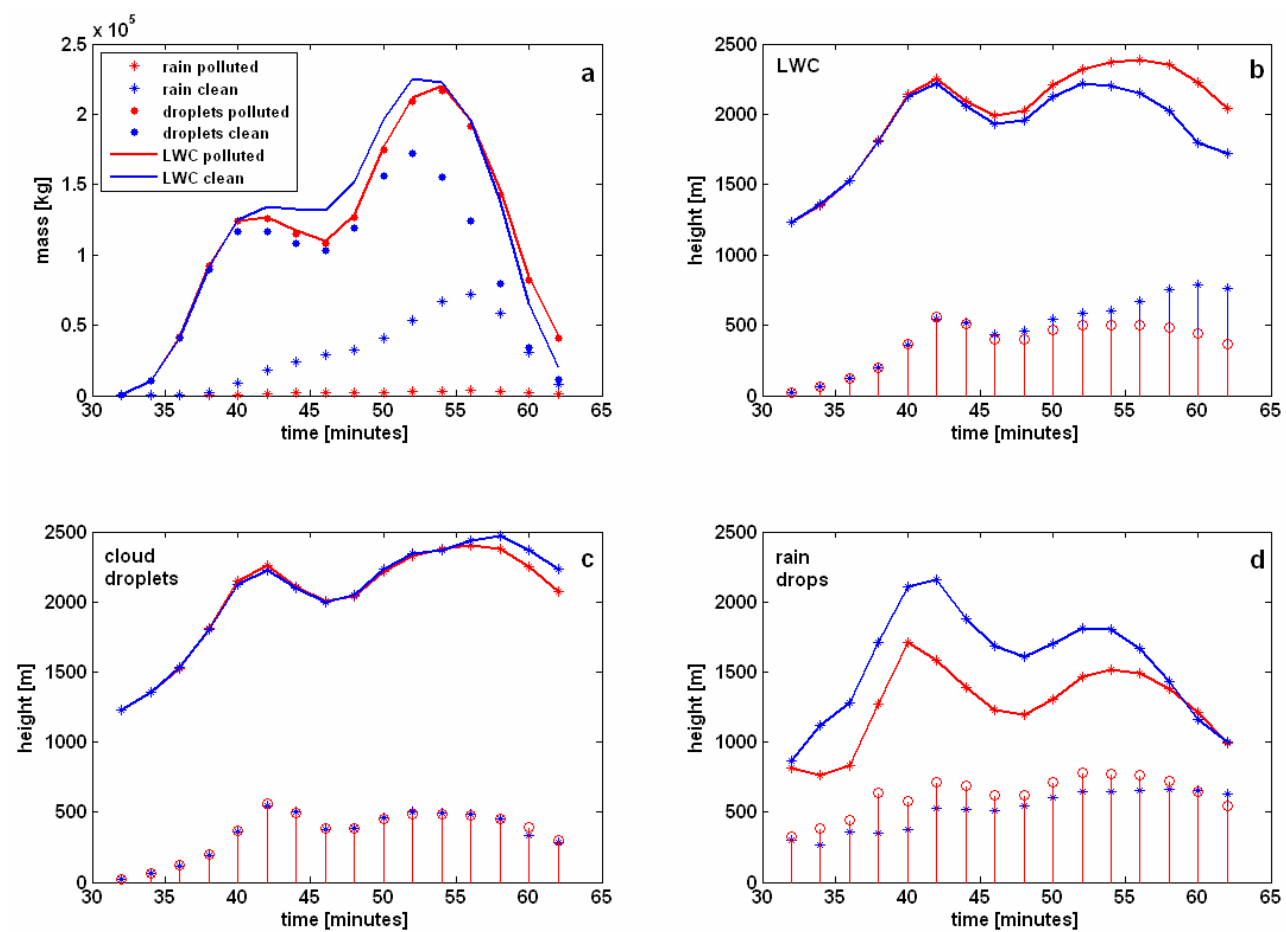

Fig. 2. Analysis of the time evolution of cloud mass for the clean (blue) and polluted (red) clouds: (a) (top, left) - total mass of the cloud liquid water (solid), cloud droplets (dots) and the rain drops (stars). (b) (top, right) - the height (curve) and the spread (in bars) of the COG total liquid water content. (c) (bottom, left) - the COG height and spread (in bars) of the cloud droplets. (d) (bottom, right) COG height and spread of the rain drops.
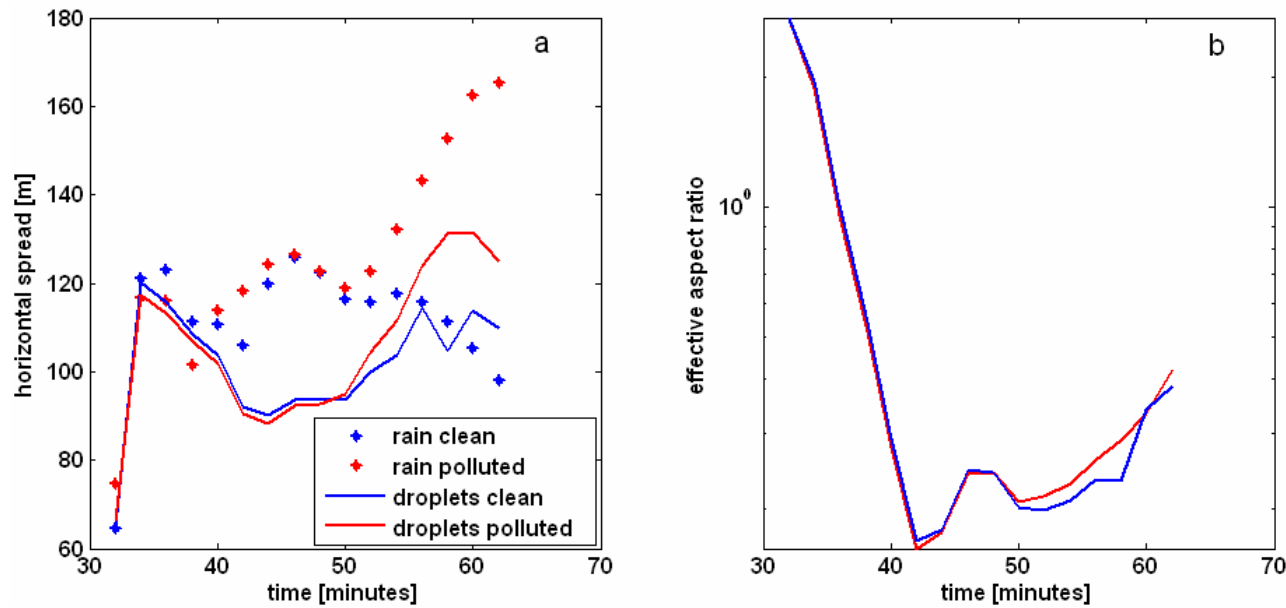

Fig. 3. (a) (left): time evolution of the horizontal spread for the cloud droplets (solid) and rain drops (stars) for the clean (blue) and the polluted (red) cloud. (b) (right): time evolution of the effective aspect ratio calculated as the ratio of the horizontal to the vertical spreads of cloud droplets for the clean (blue) and polluted (red) clouds.

better approximation of the conditions over the sea surface. The wind profile was not included in the simulations. Vertical motion was initiated in the simulations by introducing a warm bubble near the lower boundary of the model to simulate a single cloud.
The grid resolution was $50 \mathrm{~m}$ in both horizontal and vertical directions covering a three dimensional domain of $4.5 \times 4.5 \times 5.1 \mathrm{~km}$. The time step was $0.8 \mathrm{~s}$.

First, a set of cloud properties are shown (Fig. 1) using the commonly used plots of the maximum updraft velocity, maximum liquid water content (LWC), cloud-top height 
and surface rain rate. The cloud-top height is defined as the highest grid point with liquid water mixing ratio $>0.01 \mathrm{~g} / \mathrm{kg}$. Based on this set of parameters, the main differences between the clean and polluted clouds are manifested in a significantly higher rain rate for the clean cloud after about $50 \mathrm{~min}$, which depletes the LWC (Fig. 1c) and reduces the maximum updraft (Fig. 1a). The maximum cloud top height is similar for both cases (Fig. 1b).

The additional information revealed by the COG analysis is demonstrated in the next plots. The cloud hydrometeors are classified into three classes: cloud droplets, rain drops and the sum of the two classes e.g. the total liquid water content (LWC). The location of the LWC center of gravity is a weighted-by-mass average of the location of the cloud droplet COG and the rain drop COG. The development of the two clouds can be clearly described by the location of the COG, by the total mass, and the spread of the three classes. The evolution in time of the mass of the three classes (Fig. 2a) shows dramatic differences between the clean and the polluted cloud. While rain mass becomes a significant portion of the mature clean cloud liquid mass, it is insignificant for the polluted one. The vertical COG location and the spread of the total LWC are shown in Fig. 2b. Both clouds have similar COG height and spread values during the early stages of the cloud evolution (30 to $40 \mathrm{~min}$ ). Thereafter, the clean cloud COG height is markedly lower than that of the polluted cloud due to the enhanced sedimentation of the rain which pulls the rain COG downward (Fig. 2d). It is interesting to note that although the COG is almost equal in height for the droplets in the two clouds and is higher for the rain drops (Fig. 2c and d) in the case of the clean cloud, the decrease in height of the rain COG in the polluted cloud is insignificant due to its low rain mass. Thus, the height of the COG of the total LWC is higher for the polluted cloud.

One can gain more insight from the relationship between the location of the COG and the spread. The spread of the total LWC (Fig. 2b) can reflect the distance between the raindrop COG to the cloud droplet COG. The spread of the LWC in the clean cloud becomes significantly larger during the last stage of the cloud's life, when the distance between the rain drops and cloud droplets COG increases (see the blue curves in Fig. 2c, d). The spread of the cloud droplets is similar at all times for the two clouds (Fig. 2c) while the rain spread of the polluted cloud is larger for most times (though much smaller in amount - see Fig. 2a) and is concentrated lower in the cloud, suggesting drizzle-like rain.

What information can the horizontal COG and spread add? For one stationary cloud, as in this example, the horizontal COG location is not informative. However the evolution in time of the horizontal spread, for a moving cloud or clouds can give additional insight. Figure 3 a shows the evolution in time of the horizontal spread for the clean and polluted clouds. First, the spread of the cloud droplets is smaller than that of the rain drops, due to enhanced evaporation of cloud droplets at cloud boundaries. It also shows that after $50 \mathrm{~min}$ of simulation the spread of the polluted cloud becomes larger than the spread of the clean cloud, both for the cloud droplets and rain drops, suggesting that the smaller cloud droplets and the smaller raindrops in the polluted case are more affected by the horizontal advection at the higher altitudes. Figure $3 b$ follows the evolution in time of the effective aspect ratio, calculated as the ratio between the horizontal to the vertical spread of the cloud droplets (Eq. 5). It is clearly shown (for both clouds) that in the early stages of cloud development, while the vertical depth increases, the aspect ratio decreases fast. After the clouds reach their mature stage the aspect ratio increases. Note that the aspect ratio of the polluted cloud is larger during the mature parts of the simulation.

To demonstrate the power of the COG method, more standard calculations of the vertically and horizontally-integrated mass distributions are plotted for two selected times: $t=50$ min, when the clean cloud starts to rain and $t=60 \mathrm{~min}$, during the period of heavier rain from the clean cloud.

The vertical and horizontal distributions of the cloud droplet mass are similar for the two clouds at $\mathrm{t}=50$ (Fig. 4, left column, solid lines). While later at $\mathrm{t}=60$ the clean cloud has lost a significant portion of its mass to rain and has a higher base (Fig. 4, upper left panel, dotted lines) and faster decay of the mass away from the cloud center (close to the boundaries) compared to the polluted one. This is clearly reflected in the height of the droplet COG and spread (Fig. 2c and 3a). The vertical distribution of raindrop mass exhibits a shift towards a lower maximum height and more surface rain with the passage of time from $50 \mathrm{~min}$ to $60 \mathrm{~min}$. This is clearly and compactly shown in the raindrop COG height (Fig. 2d). Finally, the large differences in the horizontal spread of rain (presented in Fig. 3a) are demonstrated again here, in Fig. 4d. The horizontal distribution of the rain is similar at $\mathrm{t}=50$ and is much narrower, and concentrated at the core of the clean cloud during the heavy rain $(\mathrm{t}=60)$.

More information can be extracted by following the time evolution of the COG of the updraft and cloud droplet radius. Figure 5a shows the updraft COG weighted by the cloud droplet mass (following Eq. (8): $R_{W}=\frac{\sum_{i} r_{i} m_{i} W_{i}}{M_{W}}$ ). Such an operator can be translated to the location of the updraft center for this particular hydrometeor class and it is notable that the COG of the updraft is at a lower height in the polluted case during the mature stages of the cloud lifetime. However, Fig. $5 \mathrm{~b}$ shows that the updraft value weighted by the cloud droplet mass (Eq. 7: $W=\frac{\sum_{i} w_{i} m_{i}}{M}$ ) is stronger for the polluted cloud throughout the simulation time. Finally, the weighted averages of the cloud droplet diameter (Eq. 7: $D=\frac{\sum_{i} d_{i} m_{i}}{M}$ ) are plotted in Fig. 5c, showing the evolution of the cloud droplet size during the cloud lifecycle and expected effect of aerosol on average drop size (the polluted cloud has smaller cloud droplets). 

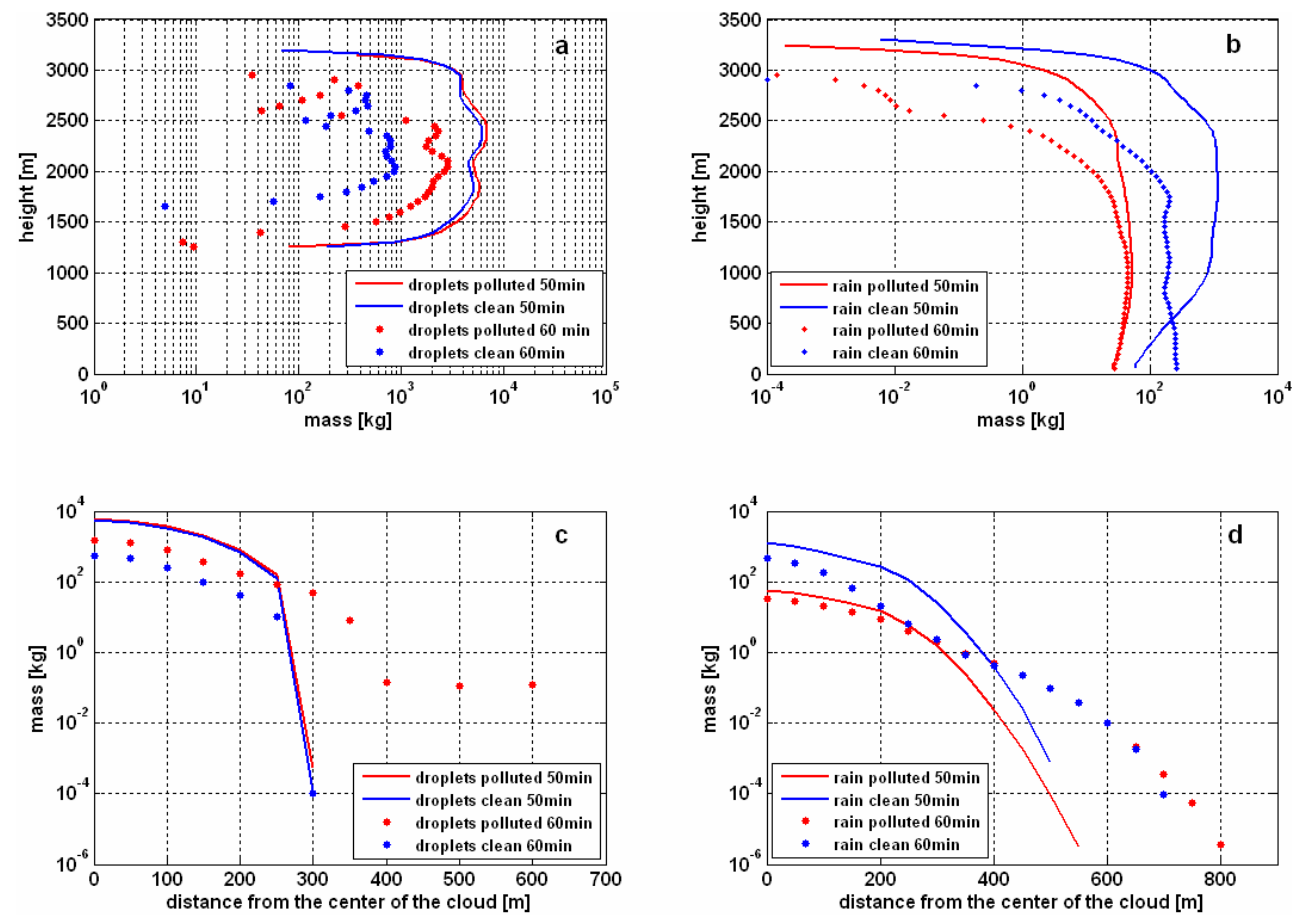

Fig. 4. Vertically and horizontally-integrated mass distributions of cloud droplets and rain drops for the clean (blue) and polluted (red) clouds. Solid lines represent distributions at $\mathrm{t}=50$ and dotted lines at $\mathrm{t}=60$. Upper row shows vertical distributions and lower row horizontal distributions. Left column shows distributions for cloud droplets and right column rain drops.
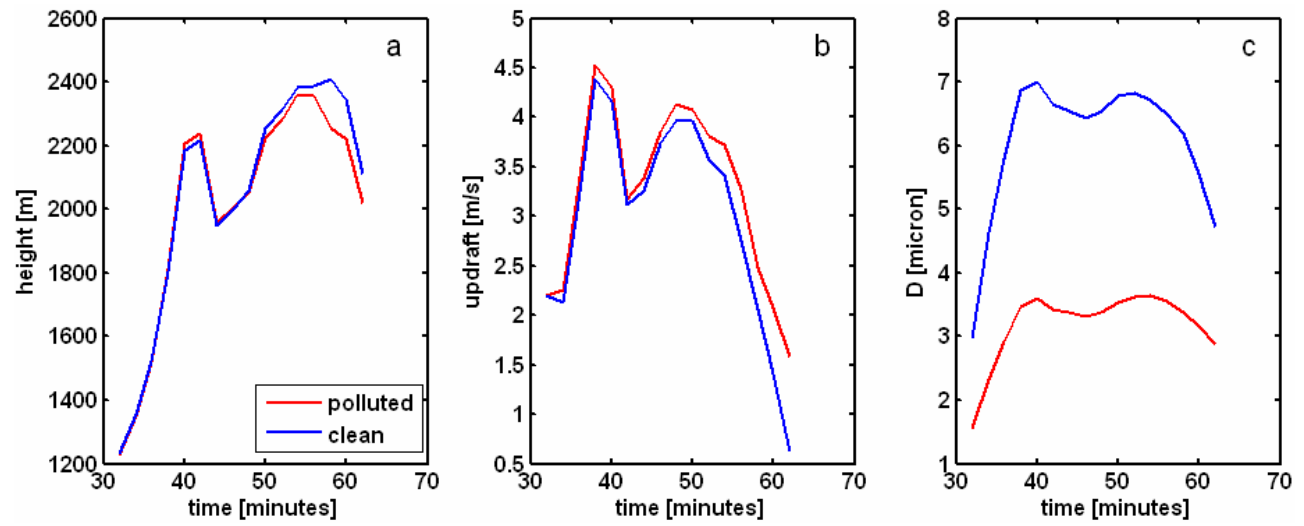

Fig. 5. (a) (left) Updraft COG. (b) (middle) Updraft weighted by cloud droplet mass. (c) (right) average radius of cloud droplets.

\section{Summary}

A novel method of analyzing cloud dynamical and microphysical properties is presented. The suggested COG method represents the clouds by the magnitude and location of the center of gravity and its spread in space. A COG-like operator is defined for cloud properties other than mass, by weighting the locations and averaging by the mass.

Such a simple representation of cloud properties in time and space allows us to gain new insight into the evolution in time and the differences between clouds of each cloud prop- erty. This method does not only reduce dramatically the dimensionality of the data, but also facilitates the interpretation of the physical processes and enhances their meaning. It provides a tool to spot interesting features in the data that later can be analyzed in depth by more detailed methods.

This method, by being statistically robust (and stable), is mostly suitable for comparison between clouds evolving in different environmental conditions or for comparison of the output of different numerical models that simulate clouds in similar conditions. 
The COG method can be applied to analysis of a cloud field (in preparation). It can show trends, distributions and sensitivity to environmental conditions of the location, mass, updrafts and effective radii of an ensemble of clouds.

In this study, we have demonstrated the potential of the method by using information from two moments only (location, average and spread). Other moments could be used to extract more details such as the symmetry of the cloud property around the COG or the existence of more then one maximum in the spatial distribution of the mass.

With the launch of CloudSat (Stephens et al., 2002) and copious amounts of other radar data, such a method can also be applied to cloud measurement data, reducing the complexity and facilitating the interpretation of physical processes in a similar manner.

Acknowledgements. This paper is dedicated to the memory of Yoram J Kaufman, a dear friend and a brilliant scientist. This research was supported in part by the Israel Science Foundation (grant 1355/06) and NASA's Radiation Sciences Program and Interdisciplinary Studies. GF was supported by NOAA's Climate Goal.

Edted by: J. Quaas

\section{References}

Cotton, W. R., Pielke, R. A., Walko, R. L., Liston, G. E., Tremback, C. J., Jiang, H., McAnelly, R. L., Harrington, J. Y., Nicholls, M. E., Carrio, G. G., and McFadden, J. P.: RAMS 2001: Current status and future directions, Meteo. Atmos. Phys., 82, 5-29, 2003.

Feynman, R., Leighton, R., and Matthew, S.: Center of Mass Moment of Inertia - The Feynman Lectures on Physics, Addison Wesley, Vol. 1, Chapter 19, ISBN 0-201-02116-1, 19-1-19-4, 1963.

IPCC: The Intergovernmental Panel on Climate Change (IPCC) website, http://www.ipcc.ch/SPM2feb07.pdf, 2007.

Levin, Z. and Cotton, W. R.: Aerosol Pollution Impact on Precipitation: A Scientific Review, WMO/IUGG International Aerosol Precipitation Science Assessment Group (IAPSAG), 485 pp., 2007.

Meyers, M. P., Walko, R. L., Harrington, J. Y., and Cotton, W. R.: New RAMS cloud microphysics parameterization. Part II: The two-moment scheme., Atmos. Res., 45, 3-39, 1997.

Stephens, G. L., Vane, D. G., Boain, R. J., Mace, G. G., Sassen, K., Wang, Z., Illingworth, A. J., O’Connor, E. J., Rossow, W. B., Durden, S. L., Miller, S. D., Austin, R. T., Benedetti, A., and Mitrescu, C.: The cloudsat mission and the a-train, A New Dimension of Space-Based Observations of Clouds and Precipitation, Bull. Am. Meteor. Soc., 83(12), 1771-1790, 2002.

Teller, A. and Levin, Z.: Factorial method as a tool for estimating the relative contribution to precipitation of cloud microphysical processes and environmental conditions: Method and application, J. Geophys. Res., 113, D02202, doi:10.1029/2007JD008960, 2008.

Walko, R., Cotton W. R., Meyers M. P., and Harrington J. Y.,: New RAMS cloud microphysics parameterization. Part I: The singlemoment scheme, Atmos. Res. 38, 29-62, 1995.

Walko, R., Cotton, W. R., Feingold, G., and Stevens B.: Efficient computation of vapor and heat diffusion between hydrometeors in a numerical model, Atmos. Res., 53, 171-183, 2000. 